\title{
TEMPO MÁXIMO DE FONAÇÃO (TMF) EM CORALISTAS E NÃO CORALISTAS
}

\author{
Debora Godoy Galdino, Amanda Souza Barros
}

Universidade do Oeste Paulista - UNOESTE, curso de Fonoaudiologia, Presidente Prudente, SP. E-mail: debigsusp@yahoo.com.br

\section{RESUMO}

O objetivo do estudo foi comparar o tempo máximo de fonação (TMF) de vozes coralistas e não coralistas. Participaram 40 pessoas, sendo 20 integrantes do coral de um curso de licenciatura em Música e 20 de participantes não coralistas. Os participantes eram de ambos os sexos com idades compreendidas entre 18 e 40 anos. A coleta de dados consistiu da execução do TMF três vezes das vogais "a" e " $i$ ", e das consoantes "s" e "z". O grupo de não coralistas apresentou TMF maior do que o grupo de coralistas, na emissão dos fonemas a,i. Na comparação entre a relação $\mathrm{s} / \mathrm{z}$ dos grupos, $80 \%$ dos coralistas apresentaram normalidade contra $75 \%$ de normalidade dos não coralistas. Concluiu-se que os tempos máximos de fonação dos sujeitos não coralistas foram pouco maiores que dos sujeitos coralistas. Tais achados podem ser justificados pela maior presença de homens no grupo de não coralistas. Sabe-se que homens apresentam a média do tempo máximo de fonação maior que mulheres.

Palavras-chave: voz, qualidade da voz, canto, avaliação da voz, distúrbios da voz.

\section{MAXIMUM PHONATION TIME (MPT) FOR CHORISTERS AND NOT CHORISTERS}

\section{ABSTRACT}

The aim of this study was to compare the maximum phonation time (MFT) of choral and non-choral voices. It was carried out with 40 people, being 20 members from the choir of the Music course and 20 non-choir participants. Participants were of both sexes between the ages of 18 and 40 years. The data collection consisted of the execution of the TMF three times from the vowels "a" and " $\mathrm{i}$ ", and the consonants "s" and "z". The group of non-choristers presented higher TMF than the group of choristers in the emission of the phonemes a, i. In the comparison between the s/z ratio of the groups, $80 \%$ of the choristers presented normality against $75 \%$ normality of the non-choristers. It was concluded that the maximum phonation times of non-chorale subjects were little bigger than those of chorale subjects. Such findings may be justified by the greater presence of men in the non-choral group. It is known that males have the mean maximum phonation time higher than females.

Keywords: voice, quality voice, song, voice evaluation, voice disorders

\section{INTRODUÇÃO}

A voz cantada é uma das manifestações mais expressivas dos caminhos da Humanidade, da identidade de um povo e do momento psicológico de um compositor, de um cantor e de sua plateia. O coro, em sua representação física sonora é o agrupamento de várias vozes para o exercício da interpretação musical por meio do canto'. Um coral é a música de vozes harmonizadas, e portanto, seu som deve refletir o bom canto de cada um dos coralistas, desenvolvido através do treino, práticas de aquecimento vocal, terapias fonológicas e hábitos pessoais sadios.

É de suma importância, a formação de especialistas da Fonoaudiologia na área dos estudos da voz como forma de refletir o avanço das pesquisas científicas e terapias utilizadas pelos profissionais em nosso País.

Como o estudo da voz humana é de domínio multidisciplinar, envolve a interação dos fonoaudiólogos com a Medicina, Psicologia, 
Acústica, Informática, dentre outras, e seu desenvolvimento exige um estudo diferenciado. A avaliação funcional da voz é realizada através de medidas respiratórias como o tempo máximo de fonação (TMF), que permite a observação quantitativa e qualitativa de um som numa única expiração.

O tempo máximo de fonação (TMF), aferido em segundos, consiste de uma medida acústica complementar e tem demonstrado sua validade entre adultos, por se tratar do tempo máximo em que o sujeito pode sustentar um som, numa só expiração, envolvendo os subsistemas fonatório e respiratório ${ }^{2}$. Trata-se de medida indireta e confiável que quantifica a ação muscular e ondulatória das pregas vocais e o fluxo de ar dos pulmões, refletindo as condições físicas e funcionais do coralista ${ }^{3}$.

A aferição do TMF possibilita verificar a habilidade da glote em se manter fechada e indicará se o coralista tem melhores condições de controlar as forças da corrente pulmonar e da laringe. Observaram que quando o TMF é reduzido (abaixo das médias), pode haver relato por parte dos profissionais da voz de ausência de sonorização na fala espontânea ${ }^{2}$.

Portanto, o esforço vocal, a pressão de ar ou a aproximação insuficiente das pregas vocais para compensar as demandas vocais podem reduzir o tempo máximo de fonação (TMF), o que não é bom.

Os valores médios do TMF na normalidade são ao redor de 14 seg para mulheres e 20 seg para os homens, sendo esta forma de medida utilizada como teste de eficiência glótica na sustentação das vogais, em frequência e intensidades habituais'.

Pessoas com técnica vocal inadequada utilizam o TMF, com diversas inspirações e muito esforço muscular, e consequentemente, quando a função fonatória estiver comprometida haverá uma redução na quantidade de ar disponível para apoiar a fonação sadia.

Assim sendo, a pesquisa entre os coralistas e não coralistas consiste da aferição dos valores de TMF, técnica a ser utilizada na avaliação de todos os participantes sendo a mesma sustentação de uma nota vocal em uma única expiração. Para esta medição são usadas as vogais /a/, /i/ e /u/ e a emissão das consoantes fricativas /s/ e /z/, um cronômetro digital e tabulação dos números. A análise do TMF é importante porque pode ser empregada no diagnóstico de distúrbios fonoaudiológicos, acompanhamento e evolução das práticas terapêuticas.

O objetivo deste estudo foi comparar a medida do tempo máximo de fonação (TMF) de vozes coralistas e vozes de não coralistas.

\section{METODOLOGIA}

Este trabalho foi submetido e aprovado pelo Comitê de Ética em Pesquisa (CAAE 31591014.6.0000.5515) e Comitê Assessor de Pesquisa Institucional (CAPI 2108) da Universidade do Oeste Paulista de Presidente Prudente - SP sendo iniciado somente após sua respectiva aprovação.

O desenvolvimento deste trabalho contou com a participação voluntária dos sujeitos que foram esclarecidos e informados sobre todos os procedimentos realizados e os objetivos do trabalho, por meio da concordância de todos os membros do coral através da assinatura do Termo de Consentimento Livre e Esclarecido TCLE. Todos os princípios éticos em pesquisa com seres humanos contidos no Art. 29ㅇ do código de ética do Fonoaudiólogo e resolução CNS 466/12 foram respeitados ao decorrer do desenvolvimento da pesquisa.

Participaram deste estudo 40 pessoas, sendo 20 dessas, integrantes do coral de um curso de licenciatura em música e 20 de participantes não coralistas. Os participantes eram de ambos os sexos com idades compreendidas entre 18 e 40 anos.

Como critério de exclusão, não participou do estudo sujeitos que apresentaram queixas vocais, dado que foi obtido em pré-entrevista. Os sujeitos que relataram queixas vocais foram orientados quanto ao tratamento e não participaram do estudo.

Após leitura, concordância e respectiva assinatura do TCLE, os participantes foram orientados quanto aos procedimentos de execução do tempo máximo de fonação (TMF). Cada participante executou o TMF três vezes das vogais a,i, e consoantes $s, z$. Cada emissão do TMF foi gravada a fim de possível conferência do tempo posteriormente. Esse TMF foi cronometrado e documentado pelo avaliador.

Após a aprovação do projeto de pesquisa os coralistas foram abordados sobre o interesse em participar da pesquisa, junto com a explicação sobre a mesma e a participação voluntária. A 
gravação da voz dos coralistas foi realizada em sala silenciosa.

Os participantes não-coralistas foram pessoas de conhecimento da própria pesquisadora, sendo aleatórios e sem vínculo com alguma instituição. Foram pessoas da família, amigas, conhecidas, próximas e também indicadas por outros amigos. O participante podia ou não ser profissional da voz. As gravações das vozes dos participantes não coralistas foram realizadas na Clínica Escola de Fonoaudiologia da Universidade do Oeste Paulista - UNOESTE.

Após as gravações, os coralistas e não coralistas que referiram alterações vocais foram orientados quanto a procura por diagnóstico e tratamento vocal. Todos os participantes foram orientados quanto às dicas de cuidados de higiene vocal.

Durante as gravações, o participante estava sentado com tronco reto, com as costas apoiadas no encosto da cadeira, braços e mãos relaxados sobre as pernas. A todos os participantes foram solicitadas as emissões das vogais $(/ a /, / i /$,$) e consoantes (/s/ e /z/) em uma$ única emissão de som, ou seja, dentro de uma única expiração, para medição do TMF de cada um deles.

Os resultados obtidos neste estudo foram apresentados em tabelas e gráficos para facilitar a análise e a apresentação. As estatísticas descritivas utilizadas para as diversas variáveis foram: média e desvio padrão, medidas máxima e mínima e porcentagens, quando apropriadas.

$\mathrm{Na}$ comparação estatística entre o tempo máximo de fonação entre os sujeitos coralistas e não coralistas foi utilizado o teste $t$ de Student com nível de significância de $p<0,05$.

\section{RESULTADOS}

Foram avaliados os Tempos Máximos de Fonação (TMF) de 40 indivíduos de idades compreendidas entre 18 e 40 anos divididos em dois grupos, sendo 20 coralistas $(40 \%$ mulheres e $60 \%$ homens) e 20 não coralistas ( $35 \%$ mulheres e $65 \%$ homens). A média de idades no grupo de indivíduos não coralistas foi de $24,1 \pm 4,9$ anos e para os indivíduos coralistas de $22,3 \pm 3,7$ anos.

Os valores dos tempos máximos de fonação dos sujeitos coralistas e não coralistas estão apresentados nas Tabelas 1 e 2.

Tabela 1. Médias dos Tempos Máximos de Fonação dos sujeitos coralistas nas vogais /a/, /i/ e consoantes /s/e /z/.

\begin{tabular}{ccccc}
\hline \multicolumn{5}{c}{ Médias dos Tempos Máximos de Fonação - Coralistas } \\
\hline Sujeitos & /a/ & /i/ & /s/ & /z/ \\
\hline 1 & 9,4 & 9,3 & 8,2 & 8,5 \\
2 & 10,9 & 12,1 & 12,9 & 9,9 \\
3 & 16,1 & 19,3 & 23,4 & 22,4 \\
4 & 20,6 & 21,5 & 31,3 & 23,9 \\
5 & 9,5 & 10,4 & 15,1 & 12,0 \\
6 & 14,0 & 14,0 & 17,8 & 20,0 \\
7 & 13,6 & 17,6 & 10,1 & 10,8 \\
8 & 13,4 & 17,6 & 17,6 & 17,6 \\
9 & 11,4 & 13,2 & 13,4 & 11,1 \\
10 & 9,9 & 10,5 & 11,1 & 14,0 \\
11 & 7,6 & 12,2 & 19,0 & 14,2 \\
12 & 13,4 & 13,7 & 13,7 & 16,5 \\
13 & 10,6 & 12,0 & 12,0 & 10,5 \\
14 & 13,4 & 14,0 & 17,4 & 17,6 \\
15 & 7,5 & 7,2 & 7,2 & 5,8 \\
16 & 10,1 & 9,6 & 18,1 & 18,1 \\
17 & 11,7 & 19,0 & 10,3 & 9,9 \\
18 & 14,2 & 15,4 & 14,9 & 14,9 \\
19 & 10,9 & 12,5 & 12,5 & 12,5 \\
20 & 14,1 & 24,5 & 18,5 & 17,2 \\
Média & $12,1 \pm 3,0$ & $14,3 \pm 4,4$ & $15,2 \pm 5,5$ & $14,4 \pm 4,7$ \\
Min - Máx & $7,5-20,6$ & $7,2-24,5$ & $7,2-31,3$ & $5,8-23,9$ \\
\hline
\end{tabular}

Colloq Vitae2017 set-dez; 9(3): 66-73. DOI: 10.5747/cv.2017.v09.n3.v211 
Tabela 2.- Médias dos Tempos Máximos de Fonação dos sujeitos não Coralistas nas vogais /a/, /i/ e consoantes /s/e /z/.

\begin{tabular}{ccccc}
\hline \multicolumn{5}{c}{ Médias dos Tempos Máximos de Fonação - Não Coralistas } \\
\hline Sujeitos & /a/ & /i/ & /s/ & /z/ \\
\hline 1 & 23,4 & 16,4 & 12,8 & 17,3 \\
2 & 19,5 & 21,0 & 17,4 & 19,9 \\
3 & 4,6 & 6,8 & 5,6 & 7,2 \\
4 & 6,5 & 7,0 & 7,6 & 6,5 \\
5 & 8,8 & 10,8 & 22,9 & 17,7 \\
6 & 14,5 & 15,2 & 12,1 & 16,1 \\
7 & 16,9 & 19,4 & 17,2 & 20,4 \\
8 & 9,8 & 12,7 & 9,5 & 7,2 \\
9 & 10,0 & 19,3 & 19,6 & 24,2 \\
10 & 18,5 & 25,8 & 19,8 & 21,1 \\
11 & 17,3 & 18,7 & 18,2 & 16,3 \\
12 & 14.5 & 16,2 & 17,5 & 17,1 \\
13 & 16,5 & 17,9 & 7,1 & 9,2 \\
14 & 12,0 & 21,4 & 19,7 & 26,8 \\
15 & 11,0 & 8,6 & 14,1 & 15,2 \\
16 & 15,2 & 11,3 & 6,2 & 8,2 \\
17 & 11,3 & 10,1 & 10,4 & 9,9 \\
18 & 7,9 & 18,7 & 9,7 & 9,8 \\
19 & 19,9 & 23,8 & 11,7 & 13,1 \\
20 & 7,2 & 8,5 & 8,8 & 8,4 \\
Média & $13,3 \pm 5,1$ & $15,5 \pm 5,7$ & $13,5 \pm 5,3$ & $14,7 \pm 6,1$ \\
Min - Máx & $4,8-23,5$ & $6,8-25,8$ & $5,8-23,0$ & $6,6-26,9$ \\
& & & &
\end{tabular}

A Comparação dos TMF entre os grupos de cantores coralistas e não coralistas em relação as médias, desvio padrão, máxima e mínima nas vogais /a/, /i/, /u/ e consoantes /s/ e /z/ podem ser observados na Figura 1.

\section{Comparação dos TMF entre os grupos}

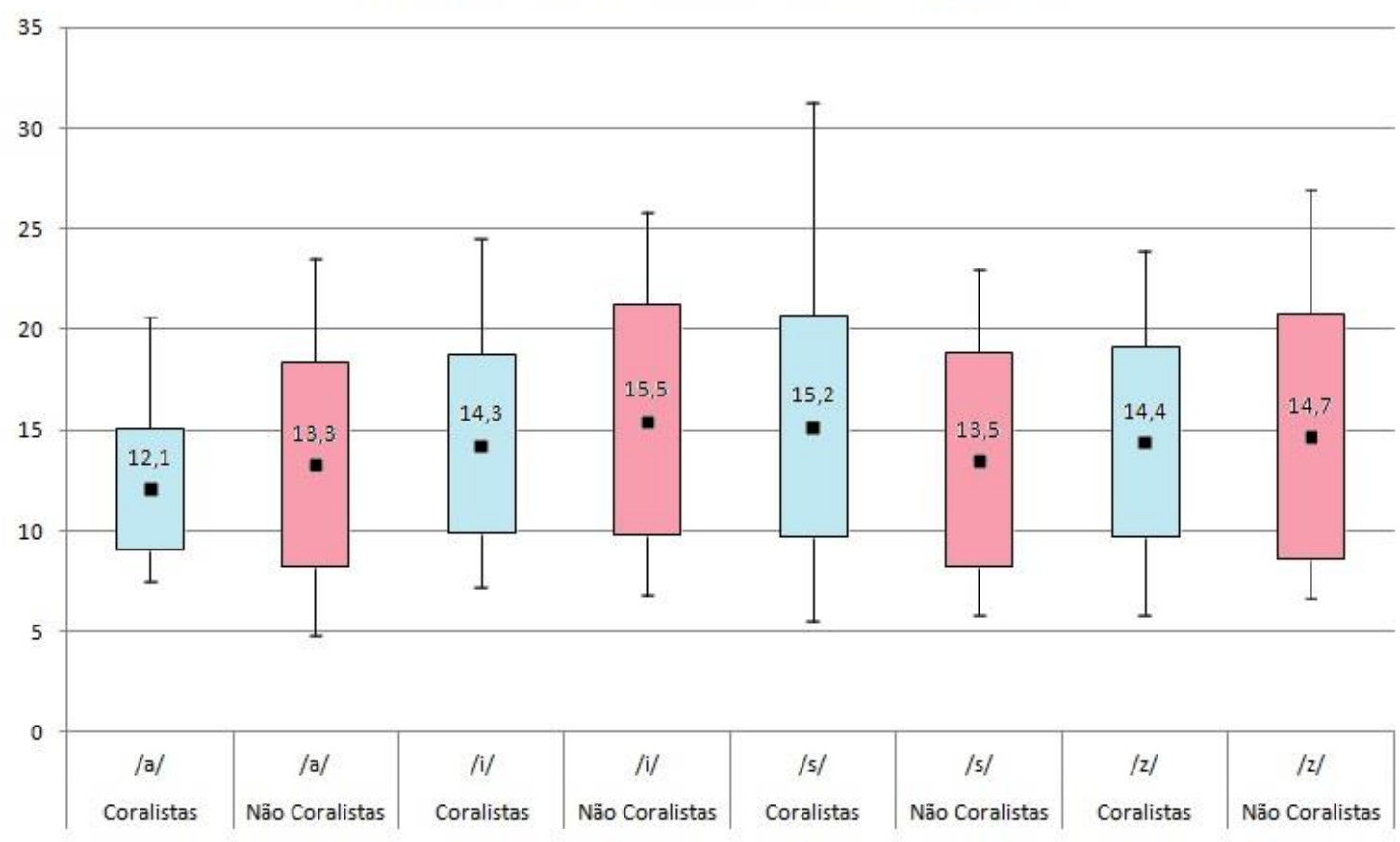

Figura 1. Comparação dos tempos máximos de fonação entre o grupo de coralistas e não coralistas. 
A relação /s/ / / z/ por sujeitos coralistas e não coralistas, assim como a interpretação sobre os valores em normais ou alterados referente à relação s/z encontram-se descritos na Tabela 3. Para a interpretação foi utilizada a referência de
Behlau (2001) que relata que valores entre 0,8 a 1,2 são normais, acima de 1,2 referem-se à falta de coaptação glótica e abaixo de 0,8 referem-se à hiperconstrição glótica.

Tabela 3. Valores da relação /s/ / /z/ por sujeitos participantes e interpretação referente à relação /s/ / /z/ dos grupos de coralistas e não coralistas.

\begin{tabular}{ccccc}
\hline Sujeitos & $\begin{array}{c}\text { Coralistas Relação } \\
\text { /s/ / / } /\end{array}$ & $\begin{array}{c}\text { Coralistas } \\
\text { Interpretação }\end{array}$ & $\begin{array}{c}\text { Não Coralistas } \\
\text { Relação / } / / / \mathbf{z} /\end{array}$ & $\begin{array}{c}\text { Não Coralistas } \\
\text { Interpretação }\end{array}$ \\
\hline 1 & 1,0 & Normal & 0,7 & Alterado \\
2 & 1,3 & Alterado & 0,9 & Normal \\
3 & 1,0 & Normal & 0,8 & Normal \\
4 & 1,3 & Alterado & 1,2 & Normal \\
5 & 1,3 & Alterado & 1,3 & Alterado \\
6 & 0,9 & Normal & 0,7 & Alterado \\
7 & 0,9 & Normal & 0,8 & Normal \\
8 & 1,0 & Normal & 1,3 & Alterado \\
9 & 1,2 & Normal & 0,8 & Normal \\
10 & 0,8 & Normal & 0,9 & Alterado \\
11 & 1,3 & Alterado & 1,1 & Normal \\
12 & 0,8 & Normal & 1,0 & Normal \\
13 & 1,1 & Normal & 0,8 & Normal \\
14 & 1,0 & Normal & 0,7 & Alterado \\
15 & 1,2 & Normal & 0,9 & Alterado \\
16 & 1,0 & Normal & 0,8 & Normal \\
17 & 1,0 & Normal & 1,0 & Normal \\
18 & 1,0 & Normal & 1,0 & Normal \\
19 & 1,0 & Normal & 0,9 & Normal \\
20 & 1,1 & Normal & 1,0 & Normal \\
\hline
\end{tabular}

As porcentagens dos valores na relação /s/ / /z/ nos dois grupos pesquisados estão descritos na Tabela 4.

Tabela 4. Porcentagem de valores na relação /s/ / /z/ nos grupos coralistas e não coralistas.

\begin{tabular}{ccc}
\hline Relação & $\begin{array}{c}\text { Coralistas } \\
(\%)\end{array}$ & $\begin{array}{c}\text { Não Coralistas } \\
(\%)\end{array}$ \\
\hline Abaixo de 0,8 & 0 & 15 \\
\hline Entre 0,8 a 1,2 & 80 & 75 \\
\hline Acima de 1,2 & 20 & 10 \\
\hline
\end{tabular}

A comparação do Tempo máximo de fonação entre os grupos de coralistas e não coralistas referentes às vogais /a/, /i/ e consoantes /s/ e /z/ estão descritos na Tabela 5.
Tabela 5. Comparação do Tempo Máximo de Fonação entre os grupos de Coralistas e Não Coralistas referentes à vogal $/ a /, / i /$ e consoantes /s/e /z/.

\begin{tabular}{lccc}
\hline & Coralistas & Não coralistas & $\mathbf{p}$ \\
\hline /a/ & 12,1 & 13,3 & 0,1032 \\
\hline /i $/ \mathrm{s} /$ & 14,3 & 15,5 & 0,6359 \\
\hline /z/ & 15,2 & 13,5 & 0,5475 \\
\hline
\end{tabular}




\section{DISCUSSÃO}

No desenvolvimento da pesquisa foram analisados os tempos máximos de fonação de dois grupos de 20 pessoas cada, sendo o primeiro grupo coralistas e o segundo constituído de sujeitos não coralistas. $O$ grupo de sujeitos coralistas eram integrantes de um coral de um curso de licenciatura em Música. Ambos os grupos se encontram em idades compreendidas entre 18 e 40 anos. O grupo de coralistas era constituído de $40 \%$ mulheres e $60 \%$ homens e o grupo de não coralistas $35 \%$ mulheres e $65 \%$ homens.

A avaliação do tempo máximo de sustentação de uma vogal é uma medida direta e confiável que tem por objetivo quantificar a relação entre a ação muscular e mucoondulatória das pregas vocais e o fluxo de ar dos pulmões, refletindo condições físicas e funcionais ${ }^{3}$. A medição dos tempos máximos de fonação visa verificar a habilidade do paciente em controlar as forças aerodinâmicas da corrente pulmonar e as forças miolásticas da laringe. Sugere, ainda, que sejam realizadas, no mínimo, três contagens de cada vogal e tomando como resultado apenas o maior valor ${ }^{3}$. Assim como descritos pelos autores, este estudo adotou as médias dos tempos maximos de fonação obtidos nas três emissões das vogais sustentadas /a/, /i/ e consoantes /s/ e /z/.

Com base nos achados encontrados, pôde-se verificar que as médias dos tempos máximos de fonação dos sujeitos não coralistas na vogal sustentada /a/ apresentou-se pouco maior do que os sujeitos coralistas. Tais achados podem ser justificados pela maior presença de homens no grupo de não coralistas. Sabe-se que homens apresentam a média do tempo máximo de fonação maior que mulheres ${ }^{1}$. Como esta medida reflete fatores de coaptação glótia e capacidade pulmonar, justifica estes achados, devido à presença da fenda triangular posterior ser fisiológica nas mulheres o que gera o escape maior de ar durante a emissão.

Capellari ${ }^{3}$ relata em seus estudos que quando o tempo máximo de fonação está alterado, tanto para valores acima da média, quanto para valores abaixo da média, podem ser indicativos de alterações na dinâmica vocal e, possivelmente, um forte indício da origem de patologias. Neste estudo, as médias encontradas no tempo máximo de fonação da vogal /a/ para coralistas foi de 12,1 seg e não coralistas de 13,3 seg. Tais valores são considerados abaixo do considerado normal que mulheres apresentam média de 14 seg na vogal sustentada ${ }^{1,4}$. O tempo máximo de fonação (TMF) estando abaixo do esperado pode ser sugestivo de incoordenação pneumofonoarticulatória, com maior escape de ar pela falta de controle neuromuscular no fechamento glótico ou, ainda, reduzida capacidade vital. Da mesma forma, TMF muito além do esperado para o sexo ou a idade do sujeito pode refletir o uso de ar de reserva, obstrução ou hiperadução glótica no momento da fonação ${ }^{3}$.

Na emissão da vogal sustentada /i/, pode ser observado que o valor do TMF aumenta nos sujeitos coralistas (média 14,3 seg) e não coralistas (média 15,5 seg) se comparados a vogal sustendada $/ a /$, sendo estes valores dentro do esperado para a média da normalidade para mulheres $^{1,4}$.

Para a fonação adequada é preciso o equilíbrio entre as forças aerodinâmicas (pulmonares) e mioelásticas (laríngeas), para que não ocorra tensão excessiva nem soprosidade em demasia'. Considera-se também que a técnica vocal inadequada leva o indivíduo a utilizar todo o TMF, gerando inspirações prolongadas, que resultam em esforço muscular; fatores tais que denotam incoordenação do mecanismo fonatório e hipertonicidade em nível glótico.

Os tempos máximos de fonação para os fricativos /s/ e /z/ são um dado importante para a relação que se pode estabelecer entre essas medidas, chamada de relação $s / z$. É relevante saber que os fonemas se dividem, quanto a sua sonoridade, em "surdos" e "sonoros". Isso significa que 0 ar expirado pelos pulmões no momento da sua execução pode ou não sofrer a ação muscular e muco-ondulatória das pregas vocais. Por exemplo, no caso dos fonemas surdos $\operatorname{com} / \mathrm{s} /$, as pregas vocais no momento da expiração estão abertas e não oferecem qualquer resistência à passagem do ar, enquanto no caso de fonemas sonoros como $/ z /$, as pregas vocais se fecham e se abrem em ciclos vibratórios ${ }^{5}$.

Mediante aos dados obtidos na relação $\mathrm{s} / \mathrm{z}$ neste estudo, $80 \%$ dos coralistas apresentaram valores correspondentes à normalidade sendo que os não coralistas apresentaram $75 \%$ de valores normais. Ainda dentro dos valores alterados, $15 \%$ dos sujeitos não coralistas apresentaram valores maiores que 1,2 o que é indicativo de falta de coaptação 
glótica e $10 \%$ dos não coralistas apresentaram valores menores que 0,8 o que é sugestivo de hiperconstrição glótica'. Entretando, no grupo de cantores coralistas, houve somente $20 \%$ de alterações nas relações $s / z$, sendo estas somente em valores acima de 1,3, ou seja, falta de coaptação glótica. Mais uma vez, estes valores corroboram com os achados encontrados nos tempos máximos de fonação reduzidos neste grupo. Tais achados podem ser justificados pela maior presença de mulheres no grupo de cantores coralistas se comparados ao grupo de não coralistas (não coralistas 35\% mulheres e coralistas $40 \%$ mulheres).

Desde o início da vida a voz é um dos meios de interação mais poderosos do indivíduo e se constitui no modo básico de comunicação entre as pessoas ${ }^{1}$. Diariamente deve ocorrer o cuidado com a voz, os profissionais devem ter percepção corporal do uso voz, independente da intensidade do seu uso, assim como há a necessidade de incorporar à rotina hábitos saudáveis para a voz que resultam em benefício próprio. Somente algumas pessoas tem consciência da importância da saúde vocal na sua profissão, já outras só conseguem perceber quando a mesma falha ou falta. Alguns sinais, tais como ardência, rouquidão, dor na garganta e pigarros frequentes podem estar relacionados com alguma patologia que acomete a laringe.

Quem utiliza a voz como ferramenta de trabalho, faz necessária a higiene vocal regularmente, mantendo hábitos saudáveis e cuidados especiais com a voz como respiração correta e o aquecimento antes de uma rotinha intensa do uso. A água também é considerada uma grande aliada da saúde vocal, pois propicia a hidratação do organismo, que refere na hidratação laríngea. Falar em ambiente ruidoso disputando com gritos são umas das situações que prejudica as pregas vocais, fazendo com que o falante grite ou aumente o tom de voz para ser ouvido, resultando em adução excessiva e brusco das pregas vocais. Este momento brusco pode causar lesões como nódulos e/ou pólipos.

Para aprender a fazer a higiene vocal adequada e manter os cuidados para não perder a voz ou desenvolver algum distúrbio dala fala, é fundamental procurar primeiro um especialista no assunto que dê as primeiras orientações corretamente.

Cantar é uma das atividades que requer ajustes específicos das estruturas envolvidas na produção da voz, para não machucar as pregas vocais. Deste modo, é de suma importância trabalhar a respiração costo-diafragma, pois é a parte responsável pela emissão da voz cantada, além de dar atenção à alimentação, aos exercícios e descanso do corpo.

Muitas pessoas que tem o "dom" de cantar tendem a achar dispensável realizar aulas de canto ou acompanhamento com um fonoaudiólogo. Elas não idealizam que além de já possuírem o privilégio da afinação, a voz necessita de cuidados especiais e até de alguns aperfeiçoamentos para que possa, assim, ser aplicada em toda sua plenitude.

O fonoaudiólogo auxiliará o cantor em alguns exercícios específicos, não interferindo na escolha das músicas, mas esclarecendo as possibilidades da voz, localizando a frequência confortável. Massagem digital na laringe depois de cantar, ou antes, de dormir, promove a vasodilatação, que possibilita maior relaxamento noturno e consequentemente, uma qualidade vocal mais satisfatória para os dias em que o uso da voz será intensivo.

Sempre beber água, alimentar-se de leguminosas e frutas, especialmente de maçã, que funciona como um adstringente natural da boca e faringe, pois além de promover um exercício de aquecimento ao mastigar, ainda retira as secreções que dificultam a vibração das pregas vocais. Estar atento à frequência da voz, evitando gritos, também auxilia nesses cuidados.

\section{CONCLUSÃO}

Diante dos resultados pôde-se concluir que, os tempos máximos de fonação dos sujeitos não coralistas foram pouco maiores que dos sujeitos coralistas. Tais achados podem ser justificados pela maior presença de homens no grupo de não coralistas, pois homens apresentam a média do tempo máximo de fonação maior que mulheres. Na relação $s / z, 80 \%$ dos coralistas apresentaram valores correspondentes à normalidade sendo que os não coralistas apresentaram $75 \%$ de valores normais, podendo ser relevados pela maior presença de mulheres no grupo de cantores coralistas se comparados ao grupo de não coralistas. Acredita-se que haja necessidade de novos estudos para que os coralistas tenham um aprimoramento vocal mais eficaz. 


\section{CONFLITOS DE INTERESSE}

Os autores declaram não haver qualquer potencial de conflito de interesse que possa interferir na imparcialidade deste trabalho científico.

\section{REFERÊNCIAS}

1. Behlau M. Voz: o livro do especialista. Rio de Janeiro: Editora Revinter; 2001.

2. Mendonça RA, Sampaio TM, Provenzano L. Medida de tempo máximo de fonação de professoras do município de Niterói/RJ. Rev. CEFAC. 2012;14(6):12048.

3. Capellari VM. Tempo máximo de fonação e características vocais acústicas de crianças préescolares. [Dissertação]. Santa Maria: Universidade Federal de Santa Maria; 2006.

4. Zemlim WR. Princípios de anatomia e fisiologia em fonoaudiologia. Porto Alegre: Ed Artmed; 2002.

5. Magalli LD, Amoroso MRM, Nishimoto IV, Barros APB, Carrara-de-angelis E. Voz, deglutição e qualidade de vida de pacientes com alteração de mobilidade de prega vocal unilateral pré e pós-fonoterapia. Rev Soc Bras Fonoaudiol. 2008;13(2):103-12. DOI: http://dx.doi.org/10.1590/S1516-

$\underline{80342008000200003}$

Recebido para publicação em 21/12/2015

Revisado em 20/06/2017

Aceito em 22/03/2018 